\title{
Bipolar Pulse Bias Effects on the Properties of MgO Reactively Deposited by Inductively Coupled Plasma-Assisted Magnetron Sputtering
}

\author{
Junghoon Joo* \\ Department of Materials Science \& Engineering, Kunsan National University, Kunsan 573-701 \\ (Received May 30, 2014, Revised May 31, 2014, Accepted May 31, 2014)
}

\begin{abstract}
$\mathrm{MgO}$ thin films were deposited by internal ICP-assisted reactive-magnetron sputtering with bipolar pulse bias on a substrate to suppress random arcs. $\mathrm{Mg}$ is reactively sputtered by a bipolar pulsed DC power of $100 \mathrm{kHz}$ into ICP generated by a dielectrically shielded internal antenna. At a mass flow ratio of $\mathrm{Ar} / \mathrm{O}_{2}=10: 2$ and an ICP/sputter power ratio of $1: 1$, optimal film properties were obtained (a powder-like crystal orientation distribution and a RMS surface roughness of approximately $0.42 \mathrm{~nm}$ ). A bipolar pulse substrate bias at a proper frequency $(\sim \mathrm{a}$ few $\mathrm{kHz})$ prevented random arc events. The crystalline preferred orientations varied between the (111), (200) and (220) orientations. By optimizing the plasma conditions, films having similar bulk crystallinity characteristics (JCPDS data) were successfully obtained.
\end{abstract}

Keywords : ICP, Bipolar pulse bias, $\mathrm{MgO}$, Reactive sputtering

\section{Introduction}

$\mathrm{MgO}$ has interesting material properties of good thermal and chemical stability as well as useful electronics properties, including an interlayer with a magnetic memory device structure while also being a high-yield secondary electron emitter. Better characteristics, such as a higher secondary electron emission coefficient, a lower outgassing rate, and a denser film structure have been pursued by researchers [1-3]. Surface contaminants of $\mathrm{MgO}$ are water-adsorbed physically and chemically [4], affecting the discharge characteristics. The surface morphology and structure are considered as important properties of $\mathrm{MgO}$ thin films. A dense structure can be obtained by proper particle bombardment during the deposition process; hence, a deposition method such as ion plating is regarded as a viable solution. $\mathrm{MgO}$ is a dielectric material. For this reason, reactive deposition starting with pure $\mathrm{Mg}$ is associated with severe surface oxidation of the Mg targets and the chamber. This can be overcome by an AC-based power technology such as pulsed DC power, which can discharge the accumulated charge on those surfaces and which can also offer a higher deposition rate than $\mathrm{RF}$ sputtering of the $\mathrm{MgO}$ target. Consequently, industries have sought large-area high-rate reactive processes with good plasma assistance. Among high-density plasmas, internal-antenna-type inductively coupled plasma can be used for this purpose. After Yamashita' s introduction of this plasma source [5], it has been assessed in various

\footnotetext{
* [E-mail] jhjoo@kunsan.ac.kr
} 
applications [6-9]. Plasma physical interpretations such as $\mathrm{E}-\mathrm{H}$ transitions, ion energy measurements, and plasma uniformity modelling have been carried out $[10,11]$. In the present study, internal-antenna-type ICP-assisted magnetron sputtering is utilized in an effort to deposit $\mathrm{MgO}$ thin films on glass substrates, and several plasma diagnostic tools are used to understand the relationship between the process parameters and the film properties. Pulsed DC sputtered films also show fairly good results with a proper substrate bias [12], but these films are less dense than those created by the ICP-assisted method [13].

\section{Experimental}

In Fig. 1, bipolar pulsed DC power is used as a sputtering source to reduce micro-arcs on the target surface. In this case, $2 \mathrm{MHz}$ ICP was used as a secondary plasma source to enhance the ionization of the sputtered $\mathrm{Mg}$ and to dissociate molecular oxygen into atomic radicals. The final practical problem associated with the

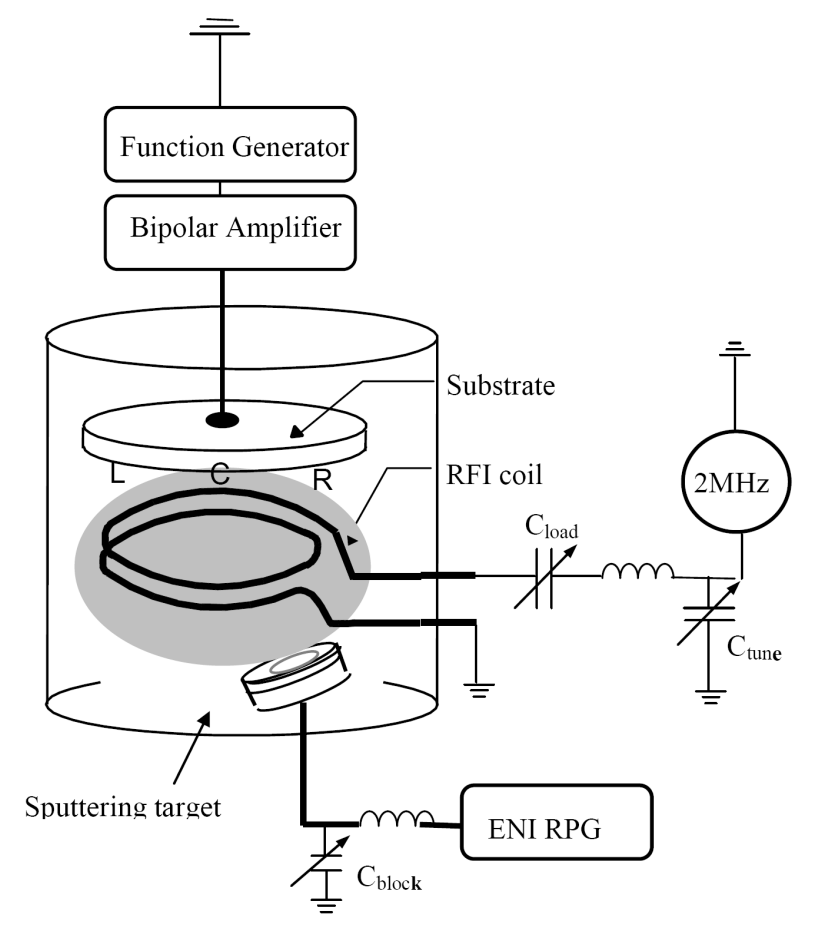

Figure 1. Experimental setup. high-density plasma process is electrical charging on the insulating substrate material, which is commonly conventional soda lime glass. Accumulated charges prevent growing film from gaining enough kinetic energy to enhance the mobility of the surface adatoms. In order to solve this charging problem and control the kinetic energy of the ions, a variable-frequency duty bipolar pulse is generated and fed into the substrate with a metal backing plate. A square pulse $\left(10 \mathrm{~V}_{\mathrm{pp}}\right)$ is generated by a pulse generator, and this signal is then fed into a bipolar operational amplifier (Kepco, BOP100-1M) and amplified to between $+20 \mathrm{~V}$ and $-40 \mathrm{~V}$. The minimum frequency to suppress abnormal discharges on the substrate was determined by monitoring the substrate voltage waveform during the deposition process. This is correlated with microstructural defects in the deposits. The crystalline structural properties of the deposited films were investigated by means of an X-ray diffraction analysis. For plasma diagnostics, CCD-based full-channel optical emission spectroscopy (OES: OceanOptics SQ-2000) was used.

\section{Results and Discussions}

\section{Substrate bias waveform: pulse frequency ef- fect on micro-arcs}

As $\mathrm{MgO}$ thin film is grown on a glass substrate, the accumulated charge often reaches an arc initiation condition, at which point the film surface becomes severely damaged. In order to reduce this type of arcing, the charge should be drained away with a proper path. A bipolar pulsed DC power feeding can be a solution, as shown in Fig. 2, which uses the frequency of the micro-arc. When the ICP power is increased, the micro-arc starting frequency also increases. This can be understood using a capacitor charging model, in which ICP acts as a charging power supply. As the charging current increases, the time to reach the threshold 

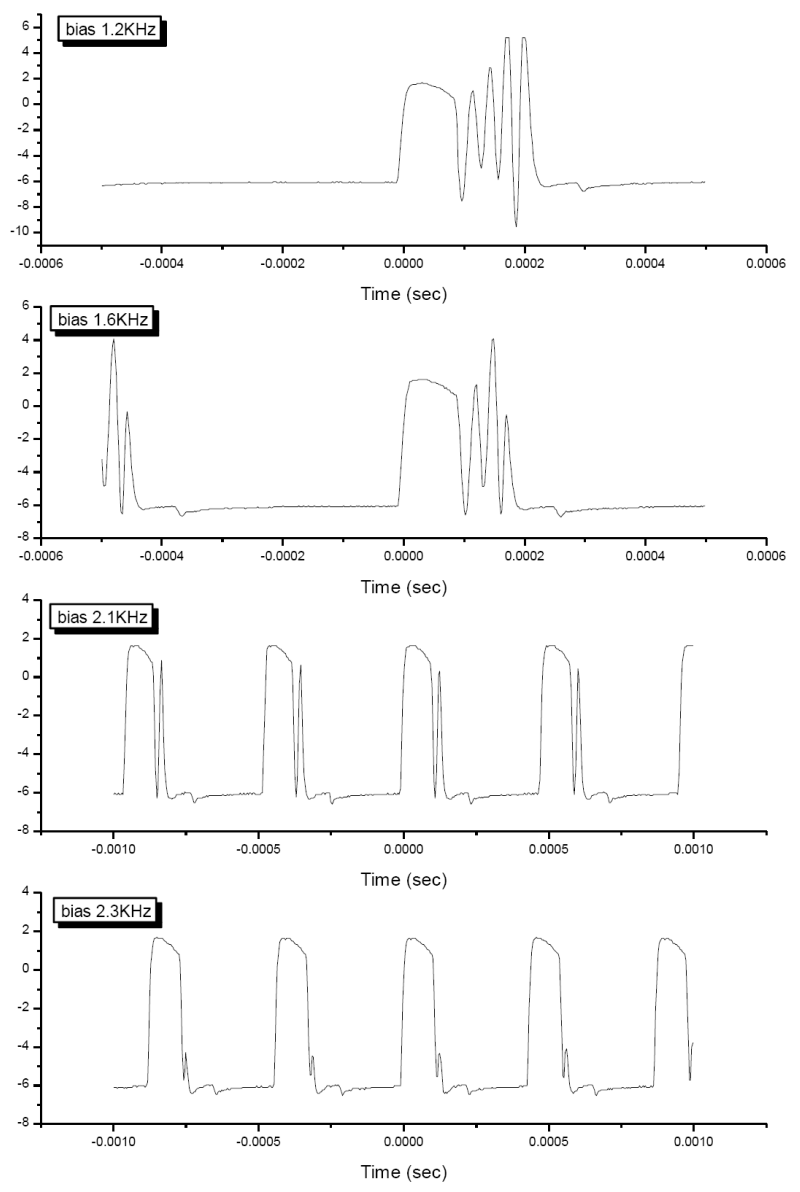

Figure 2. Bipolar pulsed DC substrate bias waveforms.

voltage of arc initiation becomes shorter. Therefore, a higher frequency of the discharge pulse is necessary. This can be an important factor to prevent defects on film surfaces. Plasma sheath evolution by pulsed DC bias can be interpreted by simple models [13]. In Fig. 3, the onset frequency of the pulsed DC bias to the substrate is increasing as the ICP power is increased. This fact can be correlated with the increased atomic oxygen radical density, as shown in Fig. 4.

\section{Plasma diagnostics: optical emission spectro- scopy}

To evaluate the number density of particles in plasma, optical actinometry is necessary. However, in many cases, it is difficult to find a proper actinomer in complex



Figure 3. Micro-arc starting frequency increases with the ICP power.

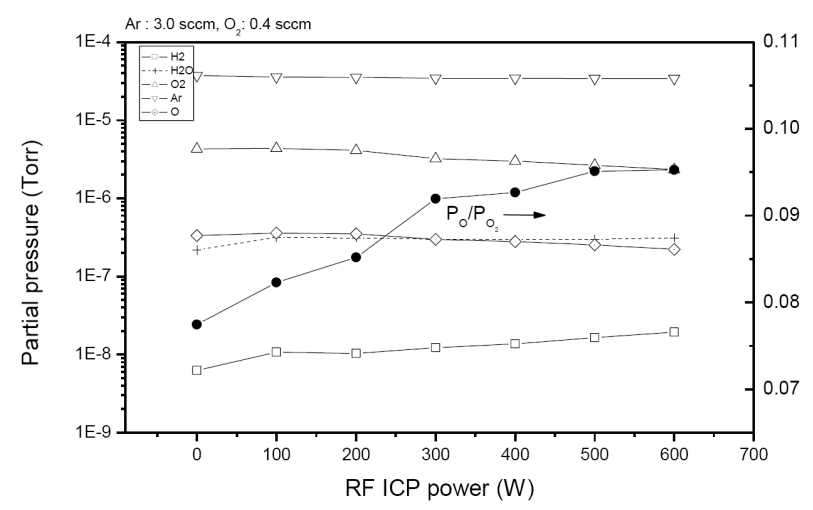

Figure 4. Dissociation ratio of $\mathrm{O}_{2}$ in ICP.

particle systems such as reactive processes. In this study, the wavelengths of $\mathrm{Mg}(729.1 \mathrm{~nm}), \mathrm{Mg}^{+}(758.0 \mathrm{~nm}), \mathrm{Ar}$ (738.3 nm), O (777.4 nm) were located as close as possible, leading to very similar effects of the plasma electrons. Usually, CCD has lower sensitivity than a PMT (photo-multiplier tube) device. However, in ICP-assisted processes, the high density of the rapid electrons in the ICP assures a high signal intensity level. In a reactive sputtering process, the ratio of the working gas to the reactive gas molecules is an important process parameter. Dissociated oxygen atoms act as radicals, and the maximum content reaches $10 \%$ in the ICP of $\mathrm{Ar}+\mathrm{O}_{2}$ (Fig. 5). From OES measurements, the emission intensities from all four species are decreasing as oxygen mixing ratio increases. This is induced by the decrease of the 


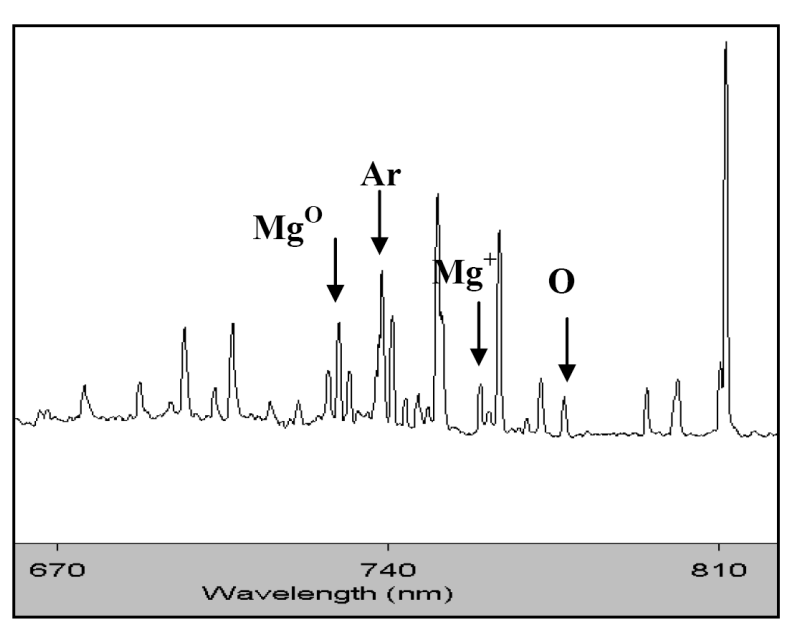

Figure 5. Optical emission spectra showing the four main particles identified.



Figure 6. OES measurements (Ar: $10 \mathrm{sccm}$, ICP : 300 $\mathrm{W}$, bias : $+20 \mathrm{~V} \sim-40 \mathrm{~V})$.

electron density as the oxygen ratio increases. When the substrate bias is applied, as shown in Fig. 6, the starting oxygen mixing ratio associated with the OES intensity drop begins to move to a higher value than normal, as shown in Fig. 7. This can be understood as evidence that the pure $\mathrm{Mg}$ deposited on the substrate is adsorbed at a rate of $100 \%$ with proper ion bombardment by the bipolar substrate bias $(+20 \mathrm{~V} \sim-40 \mathrm{~V}, 10 \mathrm{kHz})$. The normal color of the process discharge is blue in most cases, but an intense green color (possibly from $\mathrm{Mg}$ ) appears in some cases. In the OES data shown in Fig. 8, the emission intensity from $\mathrm{Mg}$ is highest and the intensity of $\mathrm{Ar}$ neutrals is a close second. As the sputtering power is maintained in a constant power mode, the measured

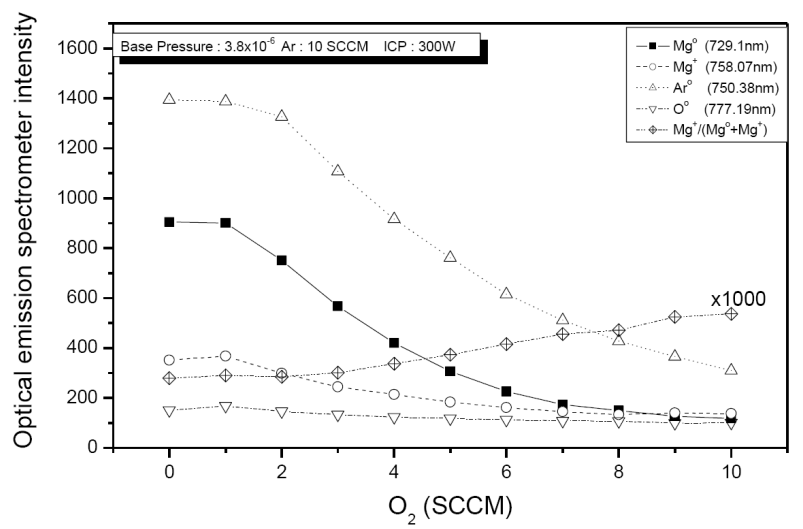

Figure 7. OES measurements (Ar: $10 \mathrm{sccm}$, ICP : 300 $W$, no substrate bias).

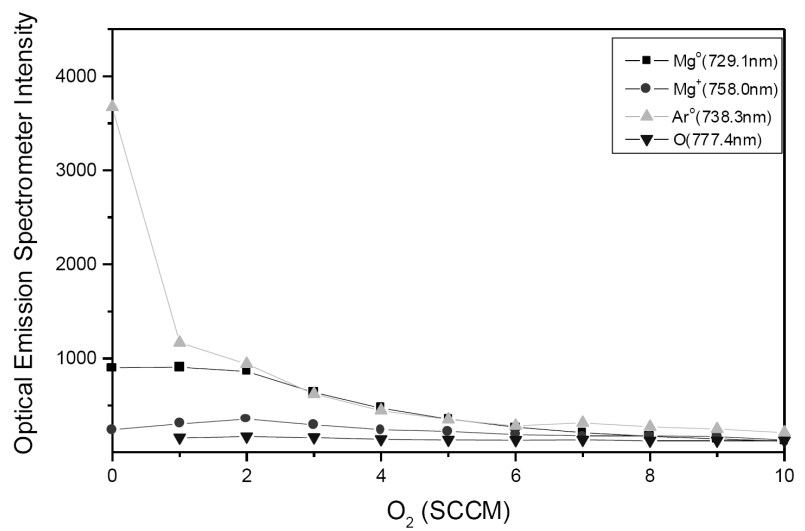

Figure 8. OES data measured when intense green color appeared.

running voltage in this condition is very low, at $150 \mathrm{~V}$.

\section{Structural and compositional properties: X-ray diffraction analysis, AFM, SEM, and ion beam analysis}

$\mathrm{MgO}$ has a $\mathrm{NaCl}$ crystal structure. Some controversy exists with regard to the preferred orientation of $\mathrm{MgO}$ thin films. We used $\mathrm{CuK} \alpha$ with a thin-film attachment with the incident angle set to 0.3 degrees. $\mathrm{MgO}$ has a $\mathrm{NaCl}$ structure, and films with the (111), (200) and (220) preferred orientations have been reported. However, the plasma erosion resistance does not coincide with the experimental results with a single crystal. This indicates that the preferred orientation of $\mathrm{MgO}$ thin film can be a 


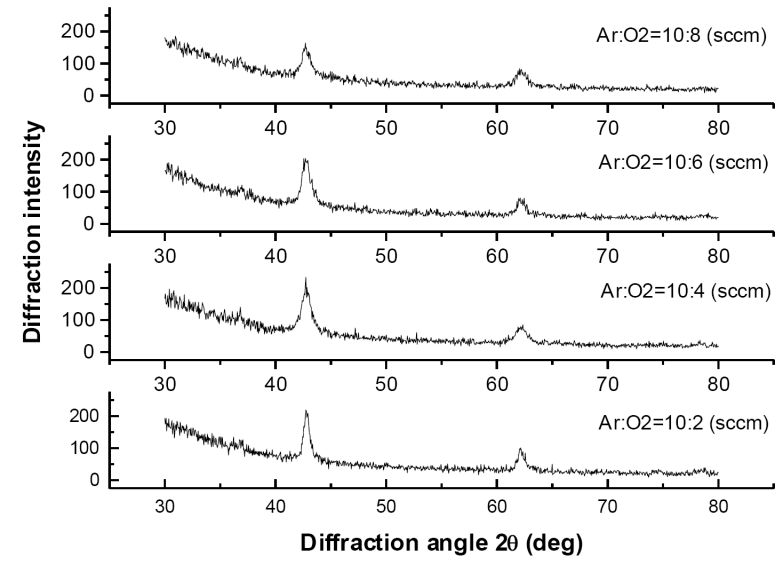

Figure 9. X-ray diffraction data showing the effects of the oxygen mixing ratio on the crystal structure.

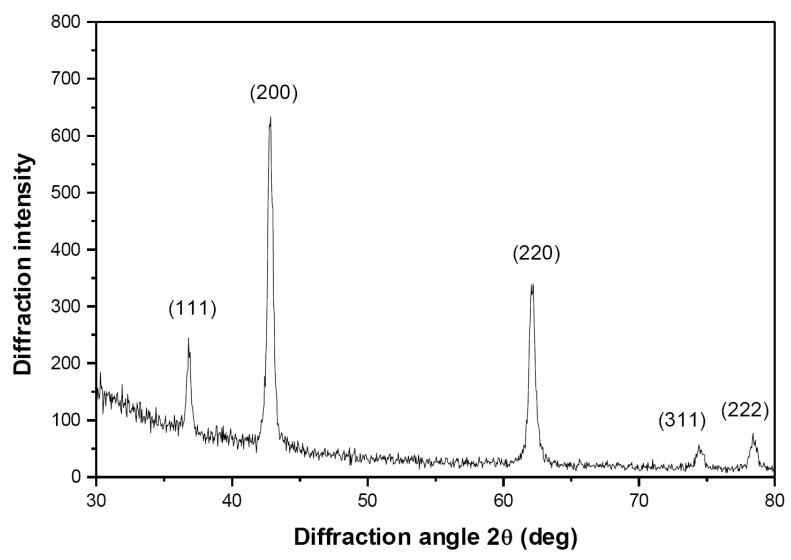

Figure 10. XRD data of MgO deposited by ICP magnetron sputtering (powder-type crystallinity $\operatorname{Ar}: \mathrm{O}_{2}=10: 2 \mathrm{sccm}, \mathrm{GMW}: 300 \mathrm{~W}$, RPG: $500 \mathrm{~W}$ ).

guideline but not an absolute test stone. In Fig. 9, even if the oxygen mixing ratio increases, the XRD pattern does not change much at this ICP power/magnetron sputtering power condition. In Fig. 10, the XRD pattern is similar to the power diffraction pattern according to the JCPDS card. Hence, with a proper substrate bias scheme (+20 V -40 V, $10 \mathrm{kHz})$, surface adatoms have enough mobility in the surface-parallel direction, a condition which does not severely develop one strong preferred orientation by resputtering. This was confirmed by AFM and SEM photos. The RMS surface roughness of the ICP-assisted magnetron-deposited sample was 0.42

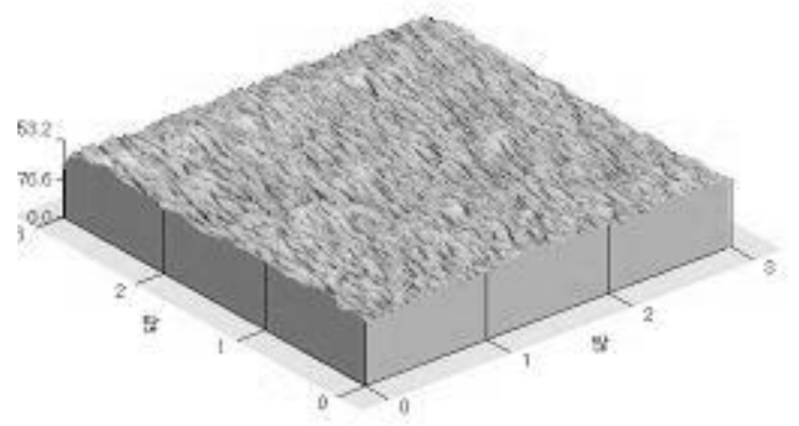

Figure 11. AFM photo of MgO: the RMS surface roughness is $0.42 \mathrm{~nm}$.
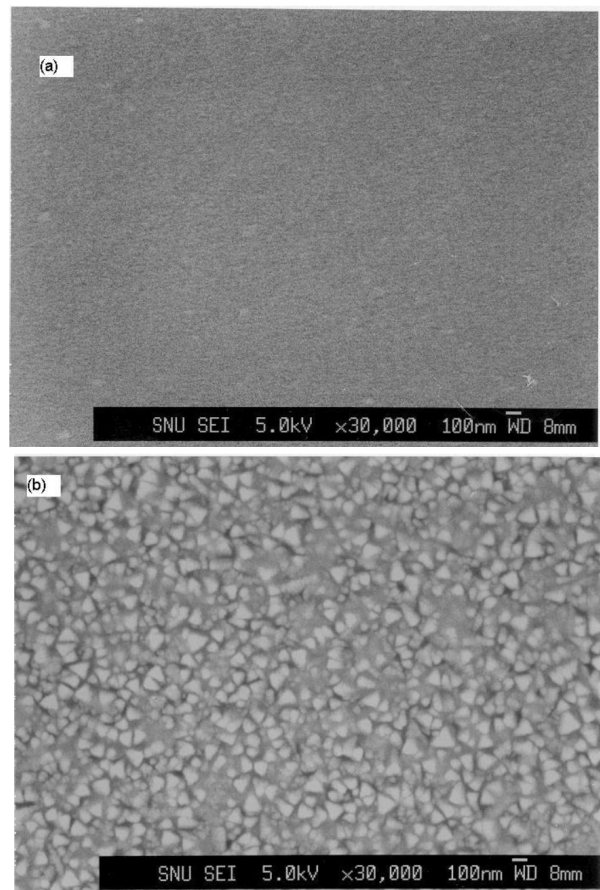

Figure 12. FESEM photo showing very smooth (a) and rough surfaces (b).

$\mathrm{nm}$ at a maximum (Fig. 11). However, the bipolar pulsed DC deposited sample has a RMS roughness $0.68 \mathrm{~nm}$ at a minimum. Moreover, the SEM photo (Fig. 12) shows a very smooth surface of the ICP-deposited films. In the pulsed DC deposited samples, sharp crystallite surfaces are shown, and the valleys are filled with contaminants (most likely $\mathrm{Mg}(\mathrm{OH})_{2}$ ). A qualitative composition analysis of the samples was conducted by means of an EPMA, and no distinct metallic components were observed. Nonetheless, the surface irradiated by medium energy $\mathrm{Ar}^{+}$ 


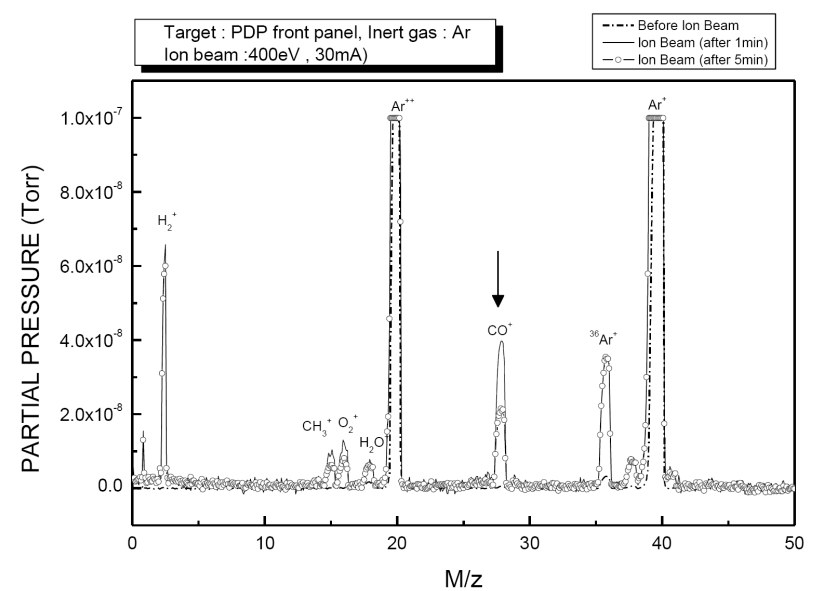

Figure 13. Ion beam analysis of an $\mathrm{MgO}$ deposited panel with $\mathrm{Ar}^{+}$beam irradiation.

showed an initial CO peak which rapidly decreased as the irradiation time increased, as shown in Fig. 13, which suggests that ICP-deposited $\mathrm{MgO}$ thin films have air-adsorbed $\mathrm{CO}$ as a main contaminant.

\section{Conclusion}

Internal-antenna-type ICP-assisted magnetron sputtering is successfully applied to reactively deposit $\mathrm{MgO}$ thin films from a pure $\mathrm{Mg}$ target. To prevent micro-arcs on the growing film surface, a bipolar pulse bias of a few tens of $\mathrm{kHz}$ was used. Optical emission investigations showed that this substrate bias affects the surface oxidation process as the oxygen mixing ratio increases. ICP-deposited $\mathrm{MgO}$ has a very smooth surface with a RMS roughness of less than $0.42 \mathrm{~nm}$, and most close-packed crystal structures show powder-like X-ray diffraction patterns. The surface observed by FESEM showed some contaminants between the crystallites in the absence of ICP assistance, and an ion beam irradiation analysis showed this is mainly CO adsorbed on the deposited $\mathrm{MgO}$.

\section{Acknowledgement}

This work was supported by the Industrial Strategic Technology Development Program (10041926, Development of High-Density Plasma Technologies for the Thin-Film Deposition of Nanoscale Semiconductor and Flexible Display Processing) funded by the Ministry of Knowledge Economy (MKE, Korea).

\section{References}

[1] M. Hashimoto, Y. Onozaki, H. Uchida, and Y. Matsumura, Rev. Sci. Instrum., 71, 999 (2000).

[2] K. H. Nam and J. G. Han, Surf. Coat. Technol. 174, 212 (2003).

[3] B. W. Byrum, Jr., IEEE Trans. Electron Devices, 22, 685 (1975).

[4] M. P. Delpanke-Ogletree, M. Ye, R. Winand, J. Mater. Res. 14, 2133 (1999).

[5] M. Yamashita, J. Vac. Sci. Technol A 7, 151 (1989).

[6] J. J. Lee and J.H. Joo, Surf. Coat. Technol. 169-170, 353 (2003).

[7] J. H. Joo, J. Vac. Sci. Technol A 18, 2006 (2000).

[8] D. K. Lee, J. J. Lee, and J. H. Joo, Surf. Coat. Technol. 173-174, 1234 (2003).

[9] B. H. Park, D. H Jung, G. R. Lee, J. J. Lee and J. H. Joo, Surf. Coat. Technol. 174-175, 643 (2003).

[10] M. Dickson, F. Qian, J. Hopwood, J. Vac. Sci. Technol. A15, 340 (1997).

[11] D. H. Kang, D. K. Lee, K. B. Kim, J. J. Lee, and J. H. Joo, Appl. Phys. Letts, 84, 3283 (2004).

[12] S. Chun and J. Baek, J. Kor. Inst. Surf. Eng. 47, 86 (2014).

[13] Y. You and J. Joo, J. Kor. Inst. Surf. Eng. 46, 168 (2014).

[14] S. Kim, H. Lee, S. park, S. Yoo, M. Cho, S. Han and B. Lim, J. Kor. Inst. Surf. Eng. 47, 53 (2014). 